\title{
TUKAMI NO KENKYÛ
}

\author{
(Dai 1 kwaino Sirase)
}

(Tsy. 15n. 4gt. 11nt. Dai 121 kwai Kôenkwai ni oite)

Seïn, Kògakusi, Nagawo-Seturô.

\section{ABSTRACT}

This peper is the first report of my practical investigations on the grab, and contains, $\mathbf{r}$. Introduction of two novel driving mechanisms of the grab of my original design, with describes of various kinds of exsisting ones, II. Investigations on the counter weight of the grab including my system, III. Investigations on the time required for one cyclic operation of the grab, and an empirical formula based on my experimental data:

$$
t=T_{t}+\left(\frac{60 \mathrm{~S}}{v}+m t^{\prime}\right)+n^{\prime} t_{h}+F_{t}
$$

where $t$, time required for one cyclic operation of grab in sec, $T_{t}$, time required for opening and closing of grab in sec., $v$, speed of rope of grab in $\mathrm{m}_{\bullet} / \mathrm{min} ., S$, total moving distance of grab in $m$., $m$, number of changes of direction of motion of grab., $t^{\prime}$, time required for breaking in sec., $t_{h}$, time required for one handling in sec., $n^{\prime}$, number of handlings., $F_{t}$, time in sec. required for miscellaneous operations not included in preceeding items.

\section{TUKAMI WO UGOKASU KIKAI.}

\section{\$1. 1-pon-duna to 2-hon-duna no Kata.}

Tukami wo zidôteki ni hatarakaseru Sikake niwa 2-tôri aru.

1) 1-pon-duna no kata:... ............ (Du 1)

2) 2-hon-duna no kata.... (Du 2) 2-tôri tomo Tuna no Kakekata ya

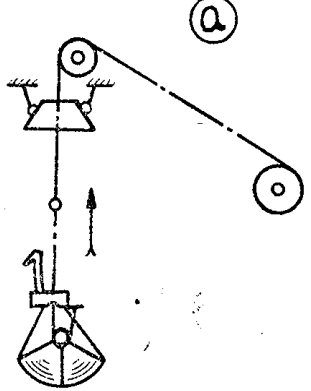<smiles>CN(C(=O)O)c1ccccc1C(N)=O</smiles>

Karakuri niwa iroiro aru ga Dôri karawa kono 2-tôri to naru. 1-pon-duna no kata wa 2-honduna no kata ni taisite Tuna no Kazu, mata, Makidô no Kazu ga hanbun de aru. Sitagatte kono hô no Karakuri ga hanbun de sumu kara kantan de aru.

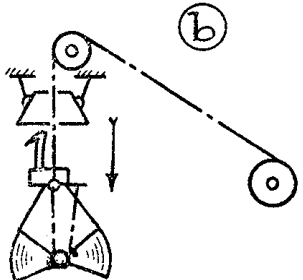

Du 1.

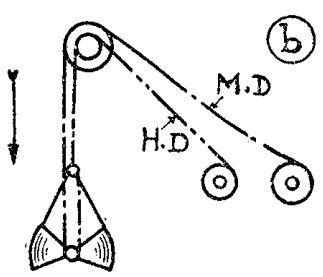

Du 2. 
Sikasi, dôsitemo 1-pon-duna de 2-hon-duna no yôni omoumama no Ugoki-kata no dekiru hazu ga nai, ima sono mazui Ten wo ageyô.

a) 1-pon-duna de Kuti wo akeru niwa Tukami zisin wo nanika ugokanai mono ni tomeru koto ga hituyô de aru kara Kuti wo akeru Tokoro (Takasa) ga kanarazu kimatte oraneba naranai koto ni naru. (Kore wo oginau niwa Kốzô ga sarani hukuzatu ni naru)

b) 1-kwai no Unten ni Handoru wo ugokasu Kazu ga 2-hon-duna no Baai no hobo 2-bai ni naru. Kono koto wa III Syô de noberu yôni Tukami no Nôryoku ni taisô hibiku no de aru. (Kono Kwankei wa tyôdo Gasu-Enzin no Baai ni yoku nite oru. 2Saikuru no Gasu-Enzin ga kyô wa mattaku tukurarezu ni tada, Diizeru-Enzin ya Semi-Diizeru-Enzin no Baai ni nomi okonawarete oru yôni, 1-pon-duna no Kata mo mata kôiu yôna Seigen wo ukeru beki mono de aru.)

\$2. Kikai no Siwake.

1-pon-duna-gata no Tukami wo ugokasu Kikai wa hutûno Maki (Hoist) de yorosii.

2-hon-duna no Tukami no Baai niwa Maki-duna wa hutûno tôri ni site, tada Hikae-duna no Toriatukai wo tokubetuni kangaereba yorosii.

Kono Sikata wo tugino 4-tôri ni suru.

a) Turiai-omori de yaru mono.

b) Tugari ni yoru mono.

c) Haguruma-zikake ni yoru mono.

d) 2-honno Tuna no sôtai-tekino Nagasa ni yoru mono.

§3. Kikai no Sikumi.

Turiai-omori de yaru mono (a) wa Du 3 ni sendu-tekini simesu tôri ni Hikae-duna wa Kusari de kono hasi ni Turiai-omori wo tukete Tuna no agari sagari ni sitagawase, Kuti wo akeru Toki niwa kono Kusari no kakaru Kusari-guruma wo hadome-suru no de, Kawa-soko wo saraeru Baai nado ni yoku tukawarete oru.

Tugari ni yoru mono (b) wa Du 4 ni simesu yôni Hikae-duna no kakaru Dô ni 1-kumi no Hadome to Tugari to wo sonae, Tukami ga Kuti wo akeru toki niwa kono Tugari wo nuite kara Hadome wo suru no de, tukamu toki mo onazi de, makiageru Toki niwa Hadome wo nuite, Tugari wo ireru no da. Mata, Du 5 (c) no yôni komiitta Karakuri mo yoku tukawarete iru.

Kono hoka Maki-duna to Hikae-duna to ni betubetuni Môtoru wo tuketa mono nado wa mottomo mazui mono de aru. 


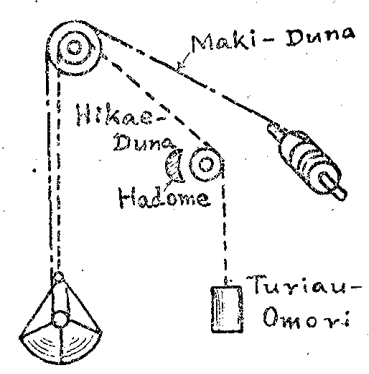

Du 3.

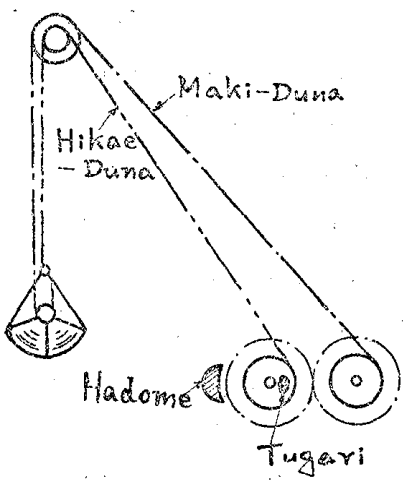

Du 4.

\$4. Kikai no Ketten.

Sate, korera no Sikake niwa tugino yôna Ketten ga aru.

1) Turiai-Omori wa Basyo no Tugo de yarinikui Baai ga ôi.

2) Kono Kata dewa Tuna wa Kusari wo tukawanai to Tugô ga warui koto mo aru.

3) $\mathrm{Du} 4$ no Sikake dewa Hadome to Tugari tono Handoru ga 2-hon iru koto.

4) mata, kono Baai ni Handoru wo toru Toki ni sukosi demo hayai-osoi ga aru to ippô no Tuna ga yurunde warui koto ni naru.

5) Du 5 no Sikake wa Kôzô ga hukuzatu de tukuru no ni Hone ga. oreru koto.

6) mata, Haguruma ga ôkute mawaru Oto ga yakamasii koto.

7) mata, Kuti wo aketa Toki ni Maki-duna ga yurumi sugiru Osore ga aru node Tukami wo otosu Toki ni waza-waza Kuti wo

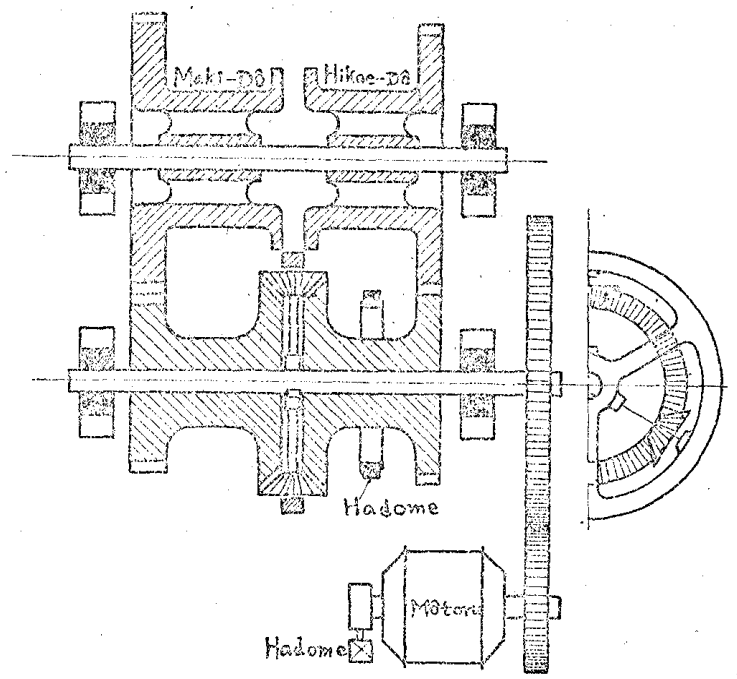

Du 5. tozite ori, tukamu Toki mata hiraku tame 2-zyûno Tema ga kakaru yôna Baai mo aru.

8) mata, Maki-duna to Hikae-duna no Tikara ga hitosiku natte hazimete makiagerareru no da kara Tukami no Kuti wo simeru Tikara ga Maki-duna ni kakaru Tikara no $m$-bai de aru toki 
ni $(m-1)$ ni heru koto ni naru. Gyakuni ieba $m$ wo masu koto wa toziru no ni iru Toki ga nagaku kakaru koto to naru.

nado de aru.

§5. Watakusi no Mokuromi (1).

Korera no ôku-no Ketten wo subete oginau tameni watasi no kangaeta Kikai ni tuite tugini noberu.

Kono Hatumei ${ }^{(1)}$ wa Makidô to Hikaedô tono sôtaitekino Ugolki wo Nezibô to Menezi no Ugoki ni totte kita mono de aru.

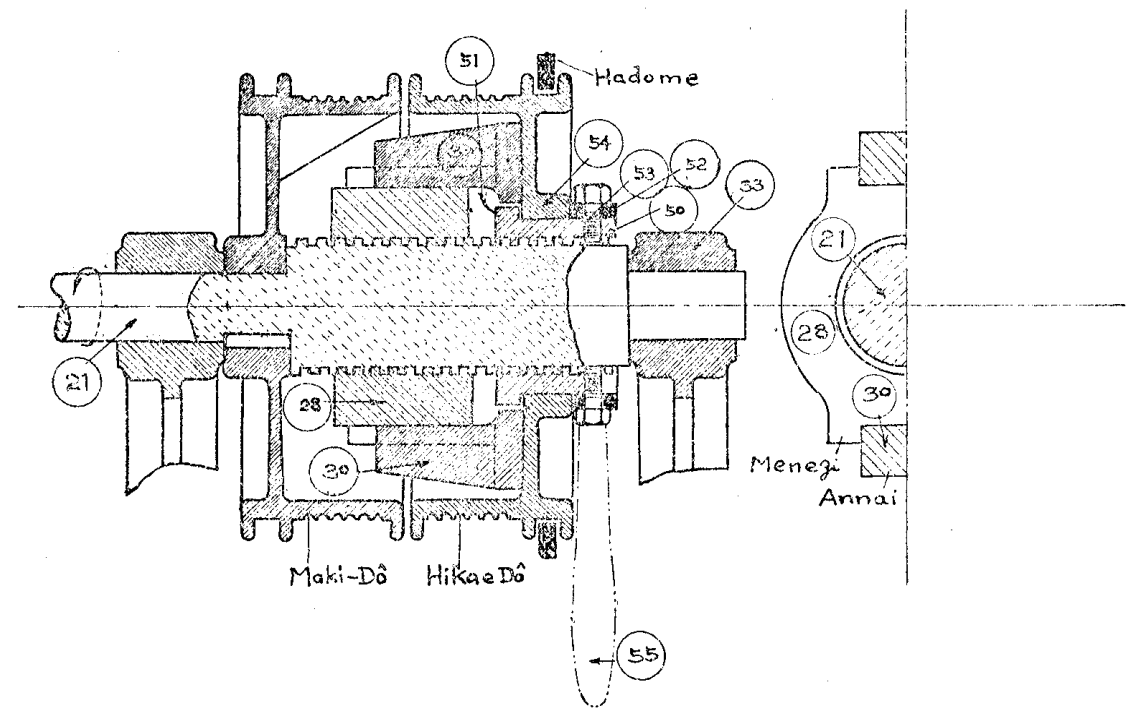

Du 6.

Du 6 ni tuite noberu to, Makidiku (21) niwa Makidô wo tomete sono saki ni Kaku-Nezi ga kitte aru. Hikaedô niwa Menezi no Annaibô (30) wo tukete Menezi (28) to tuzuku yôni suru.

Du 7 wa kono Makizuna to Hikaeduna tono sôtai-teki no Nagasa to Nezi no Hataraki to no Kwankei wo sendutekini simesita mono de aru. Hazime Tukami wo makiageru Toki niwa Menezi (kikaiteki niwa Hikaedô to kangaeru) (28) ga Makidô (23) no hô ni yotte buttukatte hitotuni natte makarete yuku, sosite Kuti wo akeru Toki niwa Hikaedô wo Hadome site Makiziku wo gyakuni mawasu kara Hikaeduna wa sonomama de Makiduna ga nobite-ḱruru kara Du no yôni Tukami wa Kuti wo akeru, sosite Menezi ga Tuba (51) ni atatta toki ni Kuti wo akekiru no de aru. Kono Makiziku wo gyakuni mawasu koto wa Tukami ni hataraku Zyûryoku ni yoru kara Menezi ga Tuba (5I) ni atatte nao osu koto de Kikai wo kowasu Sinpai ga sukosimo nai. Tugi ni Hadome

(1)Teikoku-Seihu Senbai-Tokkyo Dai 43,048. gô oyb. Dai 51,210. gô 
wo sukosi-dutu yurumeruto Tukami wa Kuti wo aketa mamade Tukamumono no ue ni otite yuku. Tugini, Tukami niwa Hadome no toreta sono mama Gendôki de Makidô wo makuto Menezi (282) wa Makidô no hô ni ututte- itte sono-mama maki-agerareru no de aru.

Koko de $\mathrm{Ki}$ wo tukeru koto wa, Menezi ga ryôhôno Tuba ni atatte buttukaru koto de, kore ga tameni Menezi to Tuba to wa hanarenu yôni sikkari kuttuite simau kara, Tukami ga Kuti wo akekitta Toki nimo tozikitta Toki no yôni Tukami zisin ni Atari (h) wo tukutte oki, kôsite Tukami no ue-sita no 2 -tu no Pin no Hedatari $L$ oyb. $l$ no $\mathrm{Na}$ gasa wo kimete oku, sosite $(L-l)$ ni sôtô-suru Menezi no Ugoki wo $A$ mata, Tuba tono Hedatari wo $L^{\prime}$ to suru to,

$$
L^{\prime}=A+a+b+\text { Menezi no Nagasa. }
$$

Kono $a$ wa Tukami ga Kuti wo tozite kara Menezi no Ikioi nomi de Makidô no hô ni susunde yuki, Hikae-duna wo yurumaseru Nagasa ni sôtôsuru Hiraki de, $b$ wa Tukami ga Kuti wo hiraite kara Menezi no Ikioi nomi de Makidô to hantai no hô ni susunde yuku node, Maki-duna wo yurumaseru Nagasa ni sôtôsuru Hiraki de aru.

$L^{\prime}$ wa kôsite kimeneba naranu ga kôiu hûni tukutte oite wa 2 -hon no Tuna ga sôtaitekini nobita toki, matawa $L^{\prime}$ no tigau betuno Tukami wo tukaitai toki niwa taisô komaru node watakusi no Hatumei ni oite wa $\mathrm{Du} 6$ ni simesu yôni Hikae-Dô no suberu Tutu (50) wo onazi Menezi ni site Nezibô ni hamekonde Tuba no Tura (51) wo hodoyoku kimete kara Nezibô (21) to kono Tutu to wo hitotuni suru tameni Osinezi (53) de tomeru no de aru. Mata, Handoru (55) wa Tutu no Tura (51) wo tekitôni naosu toki ni tukatte oru Tokoro wo simesu.

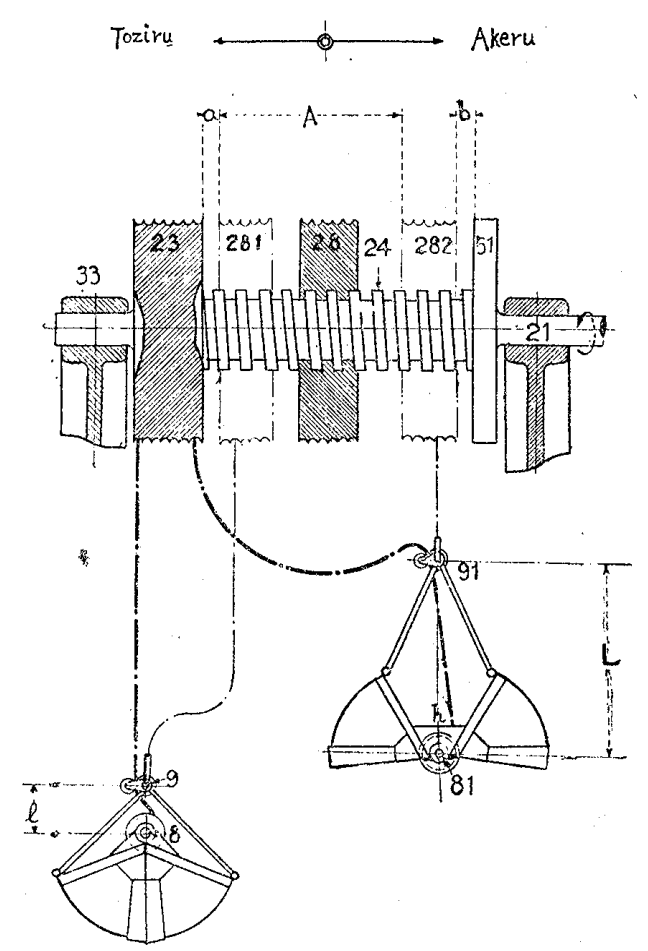

Du 7.

( $L-l)$ wa $A$ ni ataru

$a$ wa Hikae-duna no yurumi ni ataru

$b$ wa Maki-duna no yurumi ni ataru

Du 6 ni oite Nezibô to Menezi no Sikake wa 2-tu no Dô no aida ni atte iroirono Huben ga aru node, kore wo Hikaedô to Dikuuke tono aida ni oku Sekkei wo kokoromita. Du 8 no mono ni oite wa Annai- 
bô (Du 6 no (30)) wa Hilkae-Dô to tukurituke ni natte ori mata Menezi no Tuba (Du 6 no (51)) wa 2-tu ni warete oru kara tekitô no Ba ni oku ni tugô ga yoku, kore wo Bôruto de sikkarito tomeru nonimo tugô ga yoi.

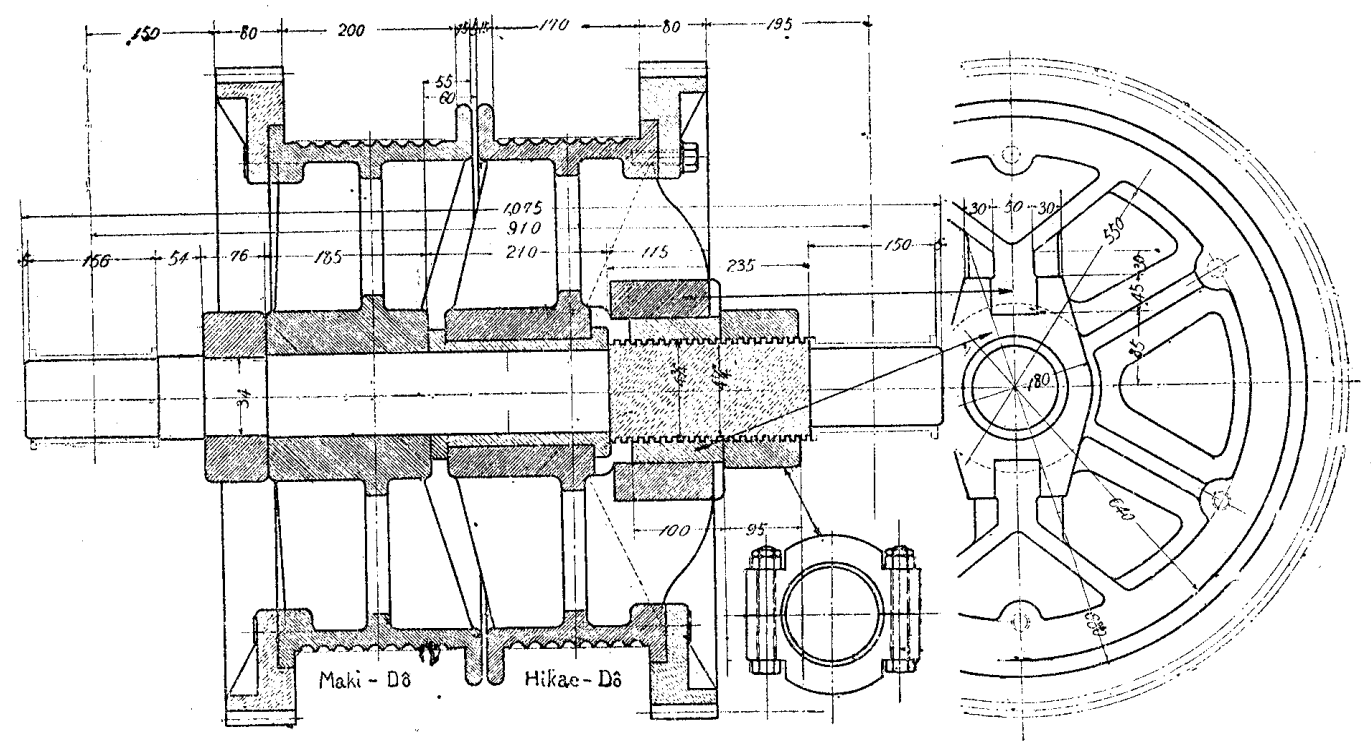

Du 8.

Nao, kono Kôan wo ôyôsite Maki-Dô ya Hikae-Dô no 2-tu aru Baai nado nimo au Sekkei wo suru koto mo deki, zissai ni kazuôku tukutte mite sôtôno Neuti wo simesita koto wo siraseru.

§6. Watakusi no Mokuromi (II).

Ue ni tokiakasita Kôan no uticle toki to site wa tugi no yôna Ten ni $\mathrm{Ki}$ wo tukeneba naranai Baai ga aruto omô.

a) Tukami wo orosu noni Tukami ni hataraku Zyûryoku to HikaeDô ni kakeru Hadome no Kagen toni yoru koto naku site zidôteki ni tugôyoku otositái koto.

b) Mata kono baai ni Tukami ga Kuti wo akekitta mama de ori kessite totyû de Kuti wo sime hazimetari suru koto no nai yôni sitai koto.

c) Tukami no Turiai-Omori ni taisite sarani tugô no yoi yôni sitai koto.

Kono Meyasu ni taisuru watakusi no Mokuromi wa tugino yôde aru.

1) Hikae-Dô wo mawasu Ziku ni Zidô-Nimotu-Hadome wo tukeru koto.

2) Hikae-Dô wo mawasu Ziku to Môtoru to turanaru Ziku towo zidôtekino Tugari de musubi-tukeru koto.

Kono Kangae wo zissai ni arawasita watakusi no Mokuromi wo Du 9 ni 
tuite tokiakasu. ${ }^{(2)}$

Makidô (11) niwa Turiai-Omori (151) wo maku Dô (110) wo sonae, mata Hikaedô (12) nimo Turiai-Omori (161) wo maku Dô (120) wo sonaeru, Môtoru (5) to Makidô (11) to wa Haguruma de tunagi, Hikaedô e tuzuku Ziku (22) niwa 2-no Sikake wo môkeru. 1-tu.wa $\S 5$. ni tokiakasita to onazi Dôri no Nezibô to Menezi (32) no Sikake de, Tukami no Kuti no Ake-tozi swt. Makidô to Hikaedô tono sôtaiteki no Ugoki wo kono Menezi (32) no Ugoki ni yotte simesi Tukami ga Kuti wo tozita toki niwa Menezi wa hidari no hasi ni uturi, Kuti wo hiraita toki niwa Du no yôni migi ni ugoite ziku (22) no tuba (33) ni atatte hitotu ni naru yôni natte oru. Nao 1-tu wa sono saki ni tuketa Zidô-Nimotu-Hadome de aru. Z.-N.-Hadome wa tuneni Kurên no Makidô ni tukeru tôri no Mono de Ziku (22) ni kitta Nezi ni tugaru Menezi (2) to $\mathrm{Ha}$ dome-guruma (21) to kara naritatie ori, Hikae-Dô wa Môtoru no gawa kara wa Môtoru no mawaru Hayasa de mawasu koto ga dekiru ga, Hikae-Duna ni kakaru Nimotu ni yotte sono otite yuku Hayasa de mawasu koto wa kessite dekinai

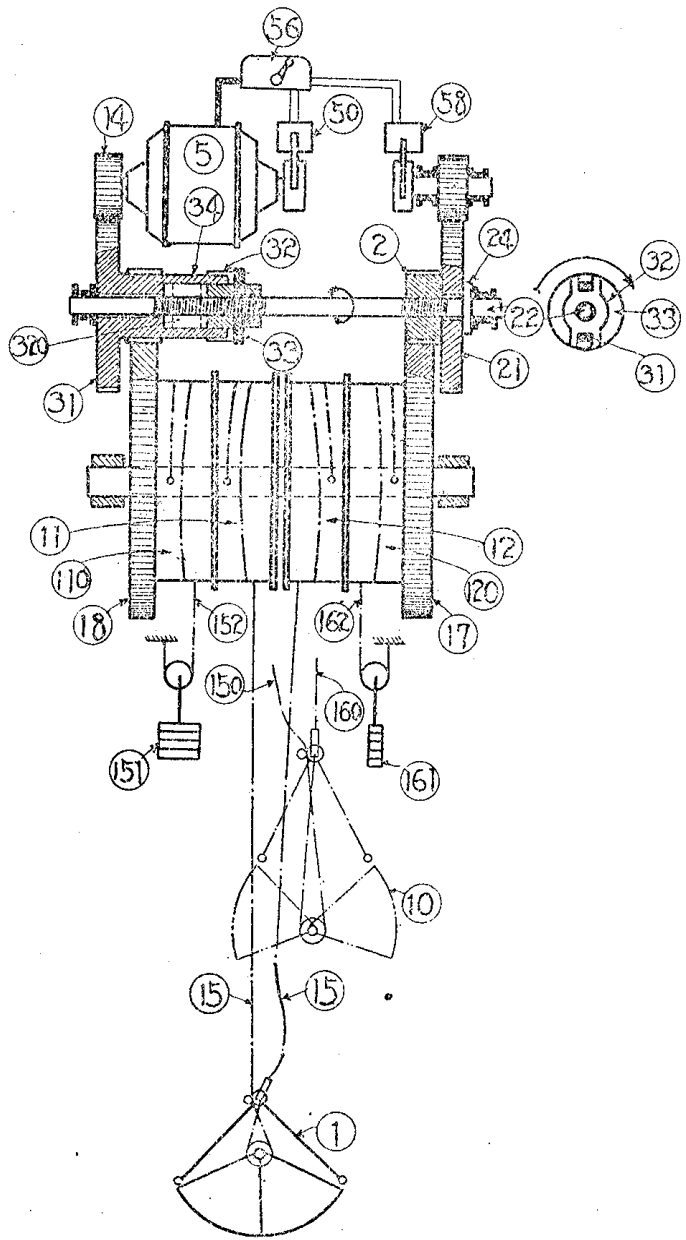

Du 9.

Sikumi de aru. Tada hitotu hutûno Mono to tigau tokoro wa, hutûno Mono dewa Hadome ni Tume wo tukatte oru ga, kono Mono dewa Denki-zikake no Hadome wo tukatte Denki-miti no ake-tozi ni yotte Hadome wo hatarakasetari hiraitari suru yôni sita koto de aru.

Sate, kono baai no Tukami no Hatarakikata wo noberuto, Tukami ga tukamu Mono no ueni aru toki ni Môtoru (5) wo maki no młki ni mawasu to (kono toki arakazime $\mathrm{Z}-\mathrm{N}-\mathrm{Hadome}$ wo toite oku) Tukami (1) wa mono wo tukami, sonomama makiagerareru. Kono toki Menezi (2)'Teikoku-Seihu-Senbaitokkyo Negai 15n. Dai 1444 gô. 
(32) wa Migi no Hasi ni ugoite yuku ga betuni ataru mono mo nai node sonomama de aru ga, Hikae-Duna (16) wa sono Turiai-Omori (161) no Hataraki ni yotte Makiduna ni sitagatte nobotte yuku no de aru. Tugini Tukami ga Tugô no yoi Ba de Kuti wo akeru niwa Z-N-Hadome no Denkimiti wo hiraite, Môtoru wo Sage no muki ni mawaseba, Hikaeduna wa $\mathrm{Z}-\mathrm{N}$-Hadome ni sasaerare Makiduna nomi yurunde Kuti wo akeru node aru kara koko de Môtoru wo tomereba yoi. Sate, Tukami ga Kuti wo ake-kiru to Menezi (32) wa Du no yôni Migi ni ututte Ziku (22) to Môtoru-gawa towo tugaraseru no de aru kara Môtoru wo Sage no Muki ni mawaseba Tukami wa Kuti wo akekiru to tomoni sonomama Hikae-Dô ni kakaru Tukami no Hûtai no Omosa no Hataraki ni yotie Kuti wo aketa mama Môtoru no mawaru Hayasa de hutatabi Mono no ueni otite yuku no de aru.

Menezi (32) no tugaru Tuba (33) wa $\S 5$. no Baai no yôni Tuna no sôtaitekino Nobi ya hokano Tukami ni taisite Arika wo kaeru yôni site aru. Mata Nezibô to Menezi to no kawarini Haguruma-zikake wo tuketemo onazi koto de ari, sarani kono kawari ni hutûno Tugari wo môketemo yoi koto wa atarimae de aru.

\section{TUKAMI NO TURIAI-OMORI.}

\section{\$7. Turiai-Omori.}

Tukami no Hûtai wa taitei sono Nakami yori omoi koto ga ooi, tatoeba, Sekitan ni tukau 1-ton no Tukami de Nakami wa 1-ton de aru noni Hûtai wa 1:5-ton mo aru. Sorede, Bariki no Sannyô wo sureba 1-Bariki de yoi mono ga $2 \cdot 5$ Bariki mo iru kara subete no Hômen ni taihenna Son de aru ga, koremade amari kono Koto ni $\mathrm{Ki}$ wo tukete oranu yô de aru.

Naze, Tukami ni taisuru Omori wo tukenai ka ? to iu ni, tugino yôna Kangae de aru.

a) Tukami ga karuku natte tukaminikuku naru.

b) Erebêtâ no Baai no yôni ue-sita de naku Yoko nimo ugoku kara Sikake ni Konnan ga aru.

c) Mata, Tuna no Kazu ga hueruto korera no Toriatukai mo hukuzatuni naru.

Konouti (a) wa tukamu mono ga katakute, tatoeba Kawazoko wo saraeru yôna Baai toka, tukamu mono ga nebatte oru Baai nado ni, tyôdo Kui wo utu Baai no yôni, hazimeni kuikomaseru yôna Baai niwa mottomo de aru ga, Sekitan ya Suna no yôna Baai niwa taisite kono 
Hituyô mo nai. Sono Wake wa, Tukami ga itido Mono no ueni tuite kara Tuna wo makuto Tuna wa makarete yuku ga zisin no Omosa de kuiitte yuku koto no Samatage wo sinai kara de aru.

\$ 8. Turiai-Omori no Omosa.

Mazu, 1-pon-duna-gata no Baai ni Tukami no Turiai-Omori wo kimeru niwa

$w=$ Omori no Omosa. $\quad W=$ Tukami no Hûtai.

$S=$ Nakami no Omosa.

to sureba, Tukami wa $W r$ kara $(W+S)$ ni kawaru kara Erebêtâ no Baai nareba, sono Bariki wo mottomo tiisaku suru tame niwa

$w-W=(W+S)-w . \quad w=W+S / 2 \ldots \ldots \ldots(1)$

to naru. Tokoro ga, Tukami no Baai niwa, Tukami ga Kuti wo akete Mono no ueni otite nao sukosi Tuna wo yurumaseru hituyô ga aru.

Kono Toki niwa Gendôki w̉a ippôno Turiai-Omori nomi wo makiage neba naranu kara kono Syunkan niwa maki-

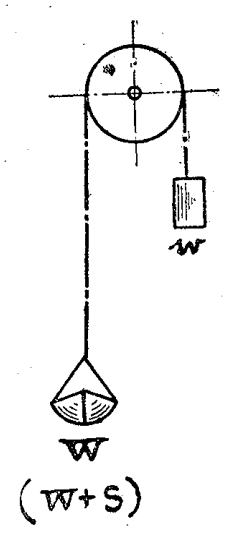

Du 10. ageru Bariki no ikubai kano ôkii Bariki ga iru no de aru kara kore dewa ikenai. Sorede, tugino Kwankei kara kimeneba naranu.

Kono Baai no Omori no Omosa wo $w v^{\prime}$ to sureba

$$
\begin{aligned}
& w^{\prime}=(W+S)-w^{\prime} \\
& w^{\prime}=\frac{W+S}{2} \ldots \ldots \ldots \ldots \ldots \ldots \ldots \ldots \ldots \ldots
\end{aligned}
$$

§9. Turiai-Omori wo tukeru Rieki.

Tukami ga Age-sage to Yoko no Yukiki tono hutatu no Ugoki wo suru baai-ni tugino yottuno Sikake ni tuite Bariki no Kawari-kata wo sirabete miru.

a) Turiai-Omori no nai Baai.

b) Turiai-Omori ga Tukami no Yoko ni ugoku Toki nimo agarisagari suru baai.

c) Turiai-Omori wa Tukami no Yoko ni ugoku Aida wa Hadome wo kakete ugokanai yôni site $w$ wo Siki (1) ni yotte kimeta Baai.

d) Ue to onazi de tada, $w$ wo Siki (2) ni yotte kimeta Baai.

Korera no Baai no Toki to Bariki to no Kwankei wo Du 11. ni simesu. Korera wa Tukami no Nakami wo 1-ton, Hûtai wo 1-5-ton, Age-sage no 
Hedatari ya* Yoko-ugoki no Hedatari wo yorosiku kime, Tuna no Hayasa wo $55 \mathrm{~m} /$ hun to site sannyô-site mita mono de aru.

Korede miru to aki-

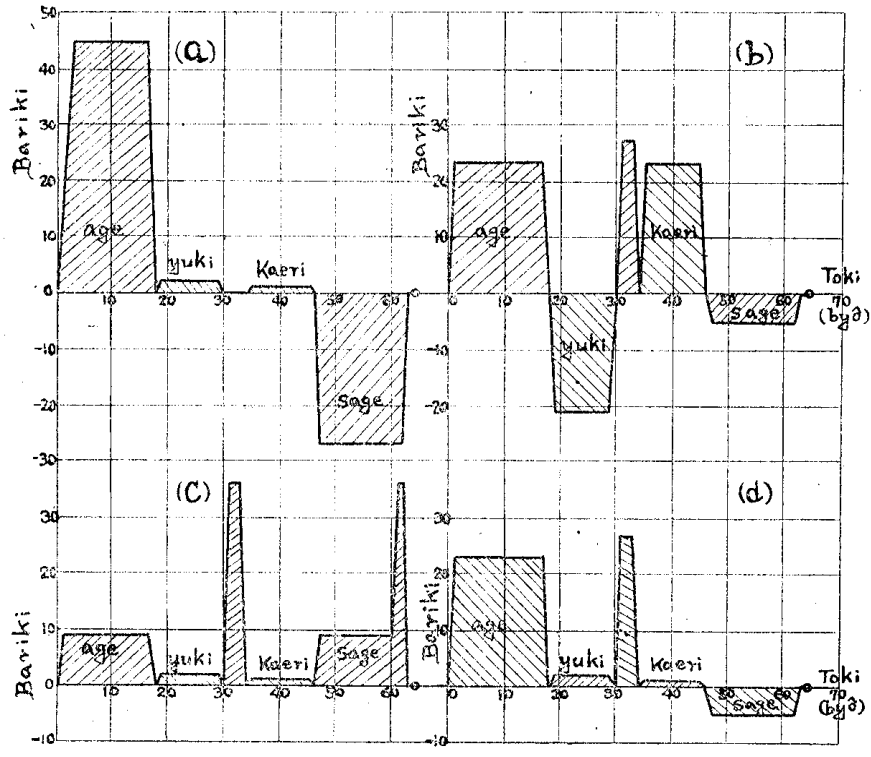

Du 11. rakana yôni, (a) wa kantan de yoi ga ôkii Bariki ga iru. (b) wa Maki-age kara Yoko-yuki ni uturu toki, mata, Yoko-yuki no totyû ni hayaku yukisugiru Sinpai ga aru kara betuni tekitôna Sikake ga iru. (c) wa Tukami no Kuti wo akeru toki to Tukami ga Mono no ueni otite nao Tuna wo yurumeru toki to ni ôkii Bariki ga iru. Sorede (d) ga itiban yoi koto ga akiraka de aru.

\$10. Koremadeno Mokuromi.

Koremade kaita yôna Wake de Tukami niwa ôkuno Baai Turiai-

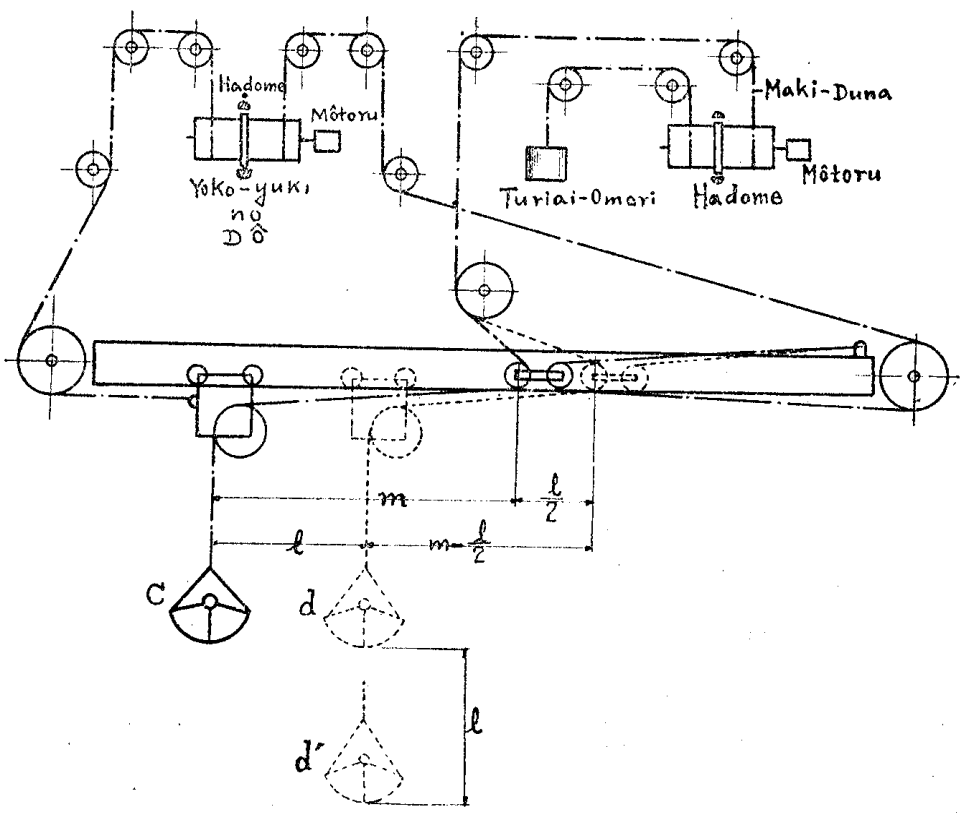

Du 12 
Omori wa tuketai no de aru ga, zissaini yaru niwa iroirono Konnan ga atte koremade okonawarete inai yô de aru.

Sikasi, Tokkyo to sitewa Igirisu Seihu no Tokkyo Dai. 12,277 (1908 n. no) ni dete oru, kore wa Du 12 ni simesu yôni Yoko-yuki no Tuna wo Yoko-yuki no Dai-Guruma no hokani Conpensating Sheave Cariers to naduketa Mono ni kakete kara Dô ni makasete ari, Maki-Duna no Dô to Turiai-Omori no Dô towa 1-pon no Diku ni toritukete aru.

Kono Sikake wa Du no ue kara wa taisô omosiroi ga, zissaino Baai niwa tugino Ketten ga aru.

a) c.-s.-c. wo motiita tameni Yoko-ugoki no Hari no Nagasa wo $50 \%$ izyô mo ato no hôni nobasanakereba naranai no de Basyo wo toru koto.

b) Basyo no Tugô ni yotte wa tôtei yarenai koto mo aru.

c) c.-s.-c. no tameni Maki to Yokoyuki no Tuna wo $180^{\circ}$ magete aru tame Tuna no Zyumyô ga mizikaku naru koto.

d) c.-s.-c. no Kuruma wo ôkiku site Tuna no Tugô wo yoku suru niwa iroiro Sekkei-zyô no Konnan ga aru.

e) c.-s.-c. no tameni Tuna no Kazu ga hueru koto.

\$11. Watakusi no Mokuromi.

Tukami no Turiai-Omori no Kake-kata ni tuite watakusi wa tugino Kôan ${ }^{(3)}$ wo sita.

$\mathrm{Du} 13$ wa kono Sikake wo Senduteki ni simesu. Maki-dô (6) Yoko-yuki no Dô (7) to Turiai-Omori no Dô (8) wo 1-pon no Ziku ni tukete, Yokoyuki no Dô (7) to TuriaiOmori no Dô (8) to niwa sorezore Hadome to Tugari to wo môkeru. Sosite Dô (7) no Hadome (16) wo toku to dôzini kono Dô no Tugari (29) ga iru. Kore to tomoni tahôno Dô (8) wo hadomesite (17) dôzini kono Dô no Tugari (20) ga nukeru yôna Sikake ni

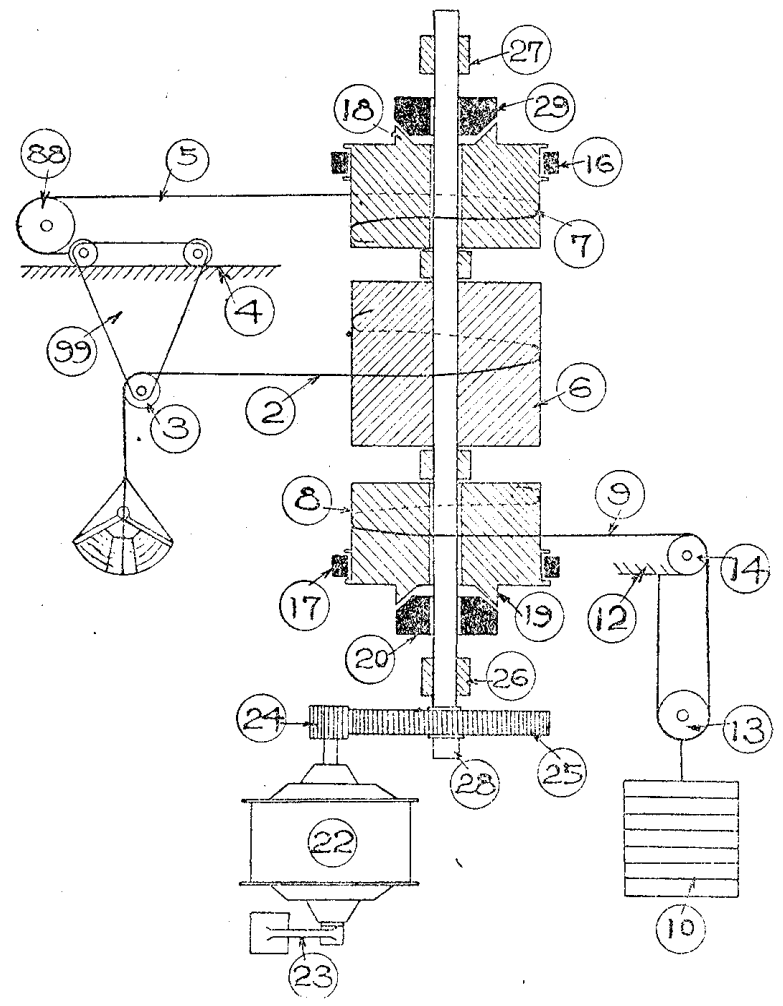

Du 13.

(3) Teikoku-Seihu Zituyô-Sinan Dai 88838 gô. 
natte oru node aru. Tukami ga Yoko ni ugoku tokiniwa Turiai-Omori ga ugokazu, mata Tukami wo maku tokiniwa yoko-yukino Dô wa Hadome site Turiai-Omori nomi ugoku no de aru.

Kono 4 no Hadome to Tugari to wa kanari hukuzatuna yô de aru ga Du 12 no Baai dewa Môtoru wo hutatu oku ueni Hadome mo iru si, mata, hitotuno Môtoru nara kore to onazi Sikake ni natta ueni maeni kaita Ketten ga aru.

Kono Sikake wa $\$ 17$ ni kaita Kikai ni ôyôsite yoi Kekkwa wo eta. (Du 31 wa kono Hadome ya Tugari wo ugokasu ziâotekino DenkiZikake wo sendutekini simesita mono de aru.)

Hutûno Turiai-Omori no nai Sikake naraba 45-Bariki jru tokoro wo kono Sikake wo tukatta tameni 25-Bariki de zyûbun de atta. Kono Baai ni okurareta Denki wa kono Kôba de zibun de okosite oru tiisai Hatudenki kara no mono de tiisai Hatudenki ni 45-Bariki to iû yôna ôkii Môtoru ga taezu ugoitari tomattari site itewa tôtei Yaku ni tatanai mono de atta. Genni 25-Bariki de sae 220-Boruto ga 150-Boruto nimo otite komatta kurai de atte, kono Sikake no Neuti wo tukuzuku kanzita no de atta.

\$ 12. 2-hon-duna-gatano Baai.

2-hon-duna-gatano Tukami no Baai ni Turiai-Omori wo môkeru niwa, Hikae-Dô to Maki-Dô to no sôtaitekino Ugoki to-wa betuni, MakiDô nomi wo Du 13 ni simesita 1-pon-Duna no Baai no Maki-Dô (6) to onaziyôni toriatukaeba yoi no de aru.

Tukami ni Turiai-Omori wo tuketa tokiniwa Tukami wo sageru tokinimo Zyûryoḱu ni joru ueni Môtoru wo Sage no Muki ni mawasu no de aru kara Môtoru ga gyakuni Omosa no tameni sono motimae no Hayasa yori masu koto no nai yôni, anzenni suru tameni Hikae-Dô wo mawasu Diku ni Didô-Nimotu-Hadome wo tukeru to yorosii. Kono koto wa $\$ 6$ no Du 9 ni simesita kara koko niwa nobenai.

Tukami no yokono Ugoki ni taisuru Sikake no komiiru Koto ni taisite wa itumo onaziyôni komaru koto de aru kara, Tukami ga yokono ugoki wo sinai demo ii yôna Kuhû wo watasi wa kangaete, hontôni kono Turiai-Omori no Sikake no Neuti wo arawasitai to kangaete oru no de aru.

\section{TUKaMi no Hataraki Ni IRU TOKI.}

§ 13. Nôryoku to Toki.

Niyaku Kikai no Nôryoku $N$ wa 1-tukami no Kasa $K$ ni 1-zikan 
ni hataraku Kazu n wo kakeawaseta mono de aru.

$$
N=n K \quad \mathrm{~m}^{3} / \mathrm{zi}
$$

1-hataraki ni iru Toki wo $t$ byô to sureba $n=\frac{60 \times 60}{t}=\frac{3600}{t} / \mathrm{zi}$.

$$
N=3600 \frac{K}{t} \quad \mathrm{~m}^{3} / \mathrm{zi}
$$

Sunawati, Nôryoku wa $K$ ni hireisi $t$ ni gyaku-hirei suru. $K$ no Ne wa tukamu Basyo no Tugô de sô ôkiku toru koto no dekinu Baai ga ôi, mata, tatoe Basyo no Tugô wa yoi ni si temo $K$ no Ne wa hituyyôna Bariki ni hireisuru kara, gizyutu-zyôno Me no Tukedokoro wa dôka site $t$ wo tiisaku suru koto de aru.

$t$ wa Tuna no Hayasa $v$ to Handoru wo toru Kazu $h_{n}$ tono Kansû to site simesareru. $t=f\left(v, h_{n}\right)$

Kono $v$ to $h_{n}$ to no utide $v$ wa Kôzan no Maki nado dewa Maku-miti ga nagai kara kanarino Hayasa ni toru ga, wareware no Mokuteki ni taisite wa Tate no Ugoki no Raai de $100 \mathrm{~m}$./hún nata, yokono Ugoki de $70 \mathrm{~m}$./hun kurai no mono de arô. Sono Wake wa mizikai tokoro, mâ, 20-mêtoru yori mizikai yôna tokoro de sô hayaku yarô to sitemo kimeta Hayasa ni naru ni iru Toki oyobi tomeru Toki ni Hadome no kiki ni iru Toki nado mo kanarino mono ni natte simau, mata, yokoni ugoite yuku Hayasa no Baai niwa kyûni tometemo Tukami wa hurete ite Kuti no akenikui koto mo aru si Untensyu no $\mathrm{Ki}$ no momeru koto mo nami taitei dewa nai.

Sokode, gizyutu-zyô mottomo Kokoro wo mukeneba naranu Mondai wa $h_{n}$ wo kazu-sukunaku suru koto de aru.

\$ 14. Tukami no Tôrimiti.

Zissai ni yoku yatte oru Baai wo Du 14 ni sendutekini simesita. $a$ de tukanda Mono wo $e$ de hakidasu no de aru. Kono Tôrimiti niwa iroiro aru.
1) $a \ldots \ldots \ldots \ldots \ldots \ldots \ldots \ldots \ldots \ldots$
2) $a \ldots \ldots \ldots \ldots b \ldots \ldots \ldots \ldots$
2) $a \ldots \ldots \ldots b \ldots \ldots f \ldots \ldots \ldots d \ldots \ldots .$.

2) oyb. 3) no Yariketa wa tyotto yoi yôni mieru, keredomo Tuna no maki-torareru Nagasa niwa Kawari ga nai no da kara tumari, Tôrimiti wa mizikaku tomo Tuna no Hayasa $v$ wo masaneba nannimo naranai si, kaette Handoru wo toru Kazu ga masu yôni naru kamo sirenai. Tugi 
ni Du' 15 no yôni Kûtyû wo Ten $b$ kara $d$ made massuguni ugokasu niwa Yoko-yuki no Tuna no Dô $D$ ni Hadome to Tugari towo sonae,

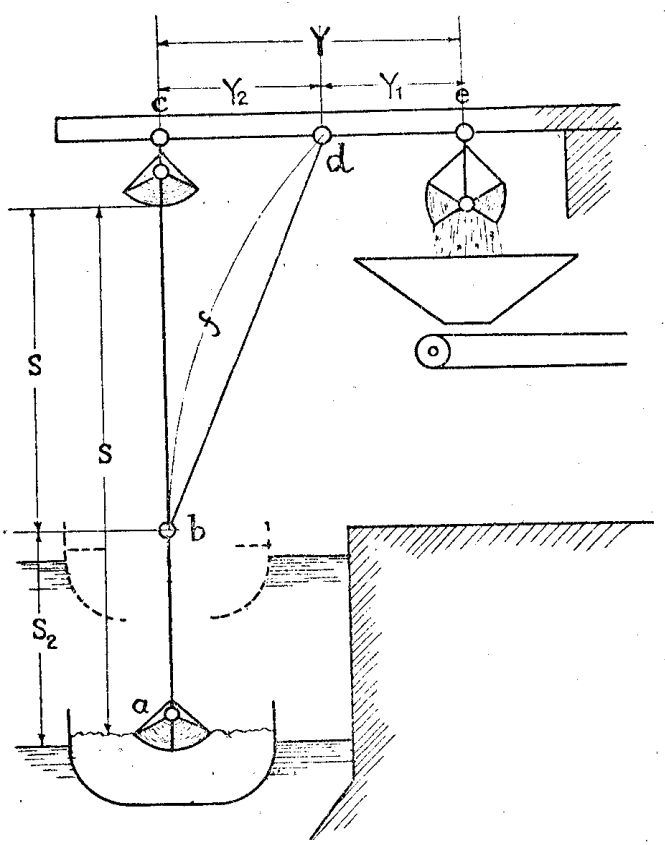

Du 14.

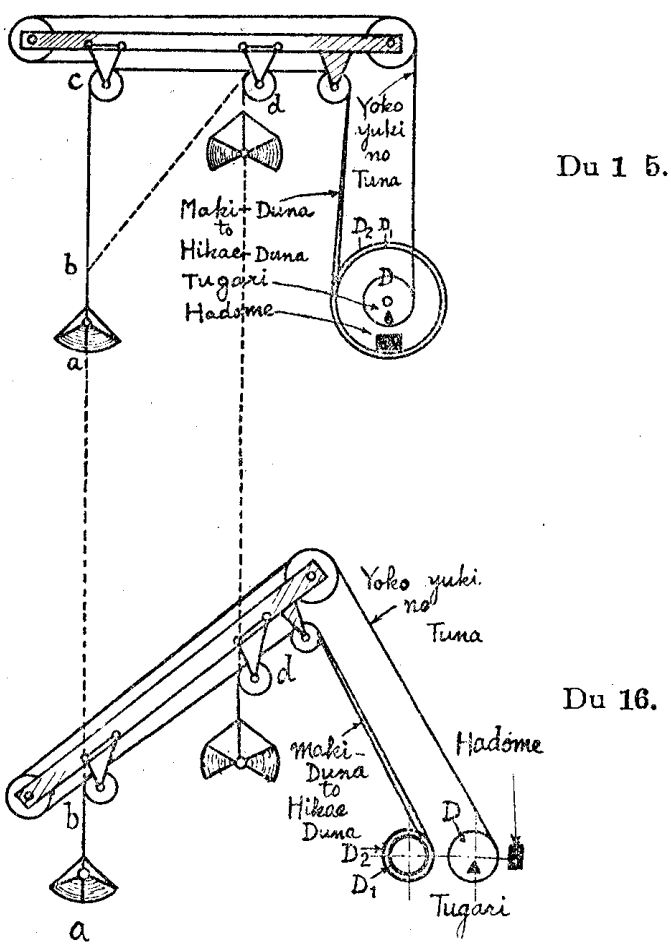

Ten $b$ de kono Hadome wo totte Tugari wo ireru no de aru. Dô $D$ to $D_{1}$ to no Kei no Wariai ga Ten $b$ kara Ten $d e$ no massuguna Ugoki wo ataeru.

Sate, kore wo Du 16 no yôni Annai-miti wo tukeru to Ten $b$ kara d ni ugokasu no ni hayai yôni kangaete iroirono Sikake wo takunande oru ga mina tumaranai, sono Wake wa,

A) Handoru no Kazu ni Kawari wa nai.

B) Yoko-yukino Tuna wo tukenai naraba, Tukami ga Ten $b$ ni todoita Toki kore ni sôtôsuru zidôtekino Sikake ģa iru.

(Tatoeba koko ni zidôtekino Hadome no Sikake wo oku yôna)

C) Ten $b$ dewa Maki-sugi no Sinpai, mata zidôtekino Sikake (B) ga umaku hataraku tameni, Tuna wo maku Hayasa wo kono Ten $b$ no mae kara kiwamete osoku seneba naranu. Sunawati, Tuna no Hayasa wo dandanni kaete yuku takumina Sikake ga itte hanahada mendô de, sorede ikkô $t$ no Ne wo tiisaku suru Yaku niwa tatanai.

\$ 15. Toki no Siwake. 
Du 14 ni tuite 1-hataraki ni iru Toki $t$ wo wakete miruto tugino yôni naru.

$t=f\left(v, h_{n}\right)$

$t=T_{t}$ (Tukami no Kuti no Ake-sime ni iru Toki)

$+U_{t}$ (Tukami no ugoku Hedatari ni yotte iru Toki)

$+H_{t}$ (Handoru wo toru no ni iru Toki)

$+f_{t}$ (Tukami no Hure wo tomeru no ni iru Toki, Untensyu

ga kangaetari magotuitari suiu no ni iru Toki nado)

$T_{t}=T_{t}^{\prime}$ (tukamu ni iru Toki)

$+T_{t}^{\prime \prime}$ (Tukami wo akeru no ni iru Toki)

$U_{t}=S_{t}($ Makiage ni iru Toki)

$+S_{t}^{\prime}$ (Makisage ni iru Toki)

$+Y_{t}$ (Yoko-yuki ni iru Toki)

$+Y_{t}^{\prime}$ (Yoko-gaeri ni iru Toki)

Sorede,

$$
t=\left(T_{t}^{\prime}+T_{t}^{\prime \prime}\right)+\left(S_{t}+S_{t}^{\prime}+Y_{t}+Y_{t}^{\prime}\right)+H_{t}+F_{t}
$$

Kore wo Sûgaku de kone-mawasitemo kekkyokuwa Yaku ni tatanai, sorede tugini. watakusi no Sekkei ni yotte watakusi ga tukutta Sikake hutatu no Rei wo agete sirabete miru koto ni suru.

§16. Hune ni tuketa Zyôki-Enzin mawasino Sekitan-Niyaku-Sôti, ${ }^{(4)}$ (Rei 1.)

Kore wa Du 17 Du 19 no Utusie ya Du 20 22 ni simesu yôna 1-ton no 2-hon-Duna-Gata no Tukami de Sekitan wo 90 ton kurai no Ki-no-Hune kara hokano Hune ni tumikaeru Yaku wo suru mono de aru. Tukami ga Hune no dono Ba nimo otoseru yôni Tukami no tôru Annai-miti wo mawasu Sikake mo tukete aru. (Koko niwa kono Koto niwa hurezuni oku)

Kikai no Hataraki no Kazu wa, Sekkei no tokiniwa, $n=100 / z i$. to sita. Dekiagatte kara Oka de yatte miruto mottomo umai Toki de 105/zi. ( $t=34$ byô) no Wariai de atta ga, kono Wariai dewa 2-nin gakari de 20-pun tuzukeru koto wa yohodono Hone ga oreta. Hune ni tukete kara no Siken dewa 90/zi. no Wariai $(t=38$ byô) de 2 -nin gakari de 30-pun ga mottomo yoi Toki de nakanaka Hone ga oreta, mata, kono Zikken wa tada $(n)$ no Kiroku wo tukurô to tutometa node, tukamu Kasa ya Sekitan no koboreru koto nado wa amari kaeri-minakatta.

(4) Tsy. 11 n. 5 gt. dekiagaru. 


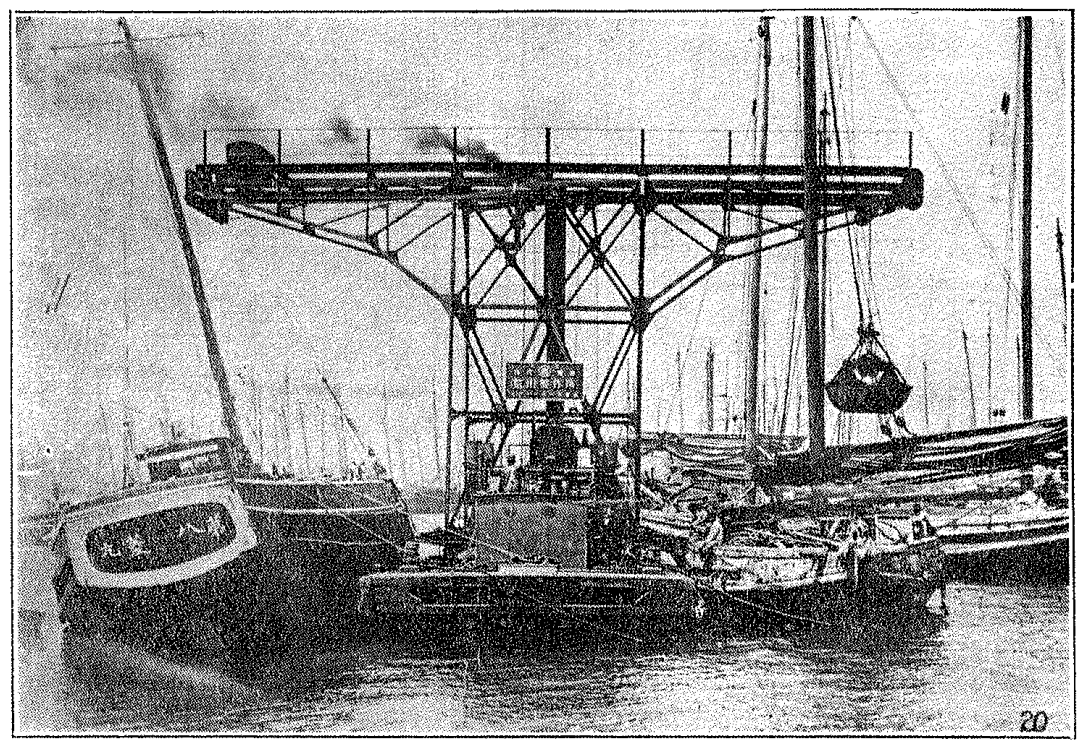

Du 17.
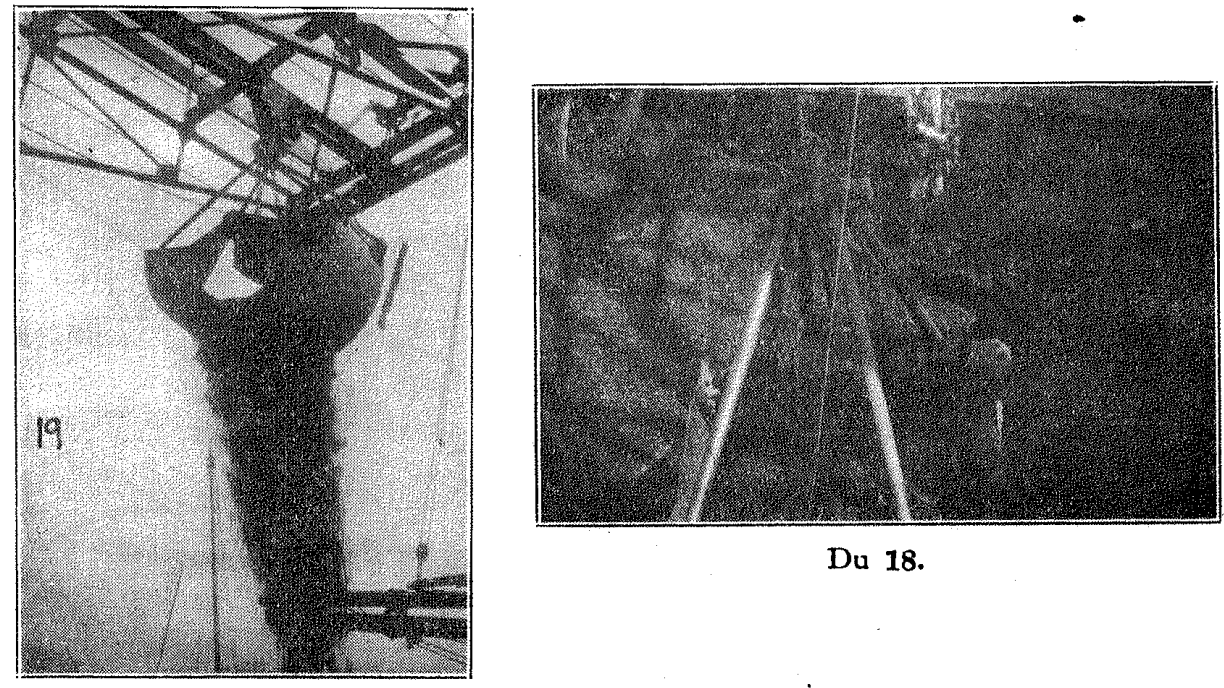

Du 18.

Du 19.

Du 23 wa kono Sôti no Handoru no Narabe-kata to Hatarakikata narabini Tukami no Arika to Arisama to wo hitomeni simesu E de aru.

Kore wa kessite Dôraku hanbunni kaita mono dewa naku, dô sureba $t$ wo mottomo tiisaku dekiru ka wo kenkyûsuru tameni iroironi kakinaositewa yatte mita saigono mono de aru.

Du no naka de 9,9 , toka $12,12,12$, toka 1 -doni 2 mo 3 mo kaite aru no wa Migi to Hidari to no Te toka, Asi to Te toka, Untensyu to Tetudaite toka iu hûni 1-doni hataraku koto wo simesu. 


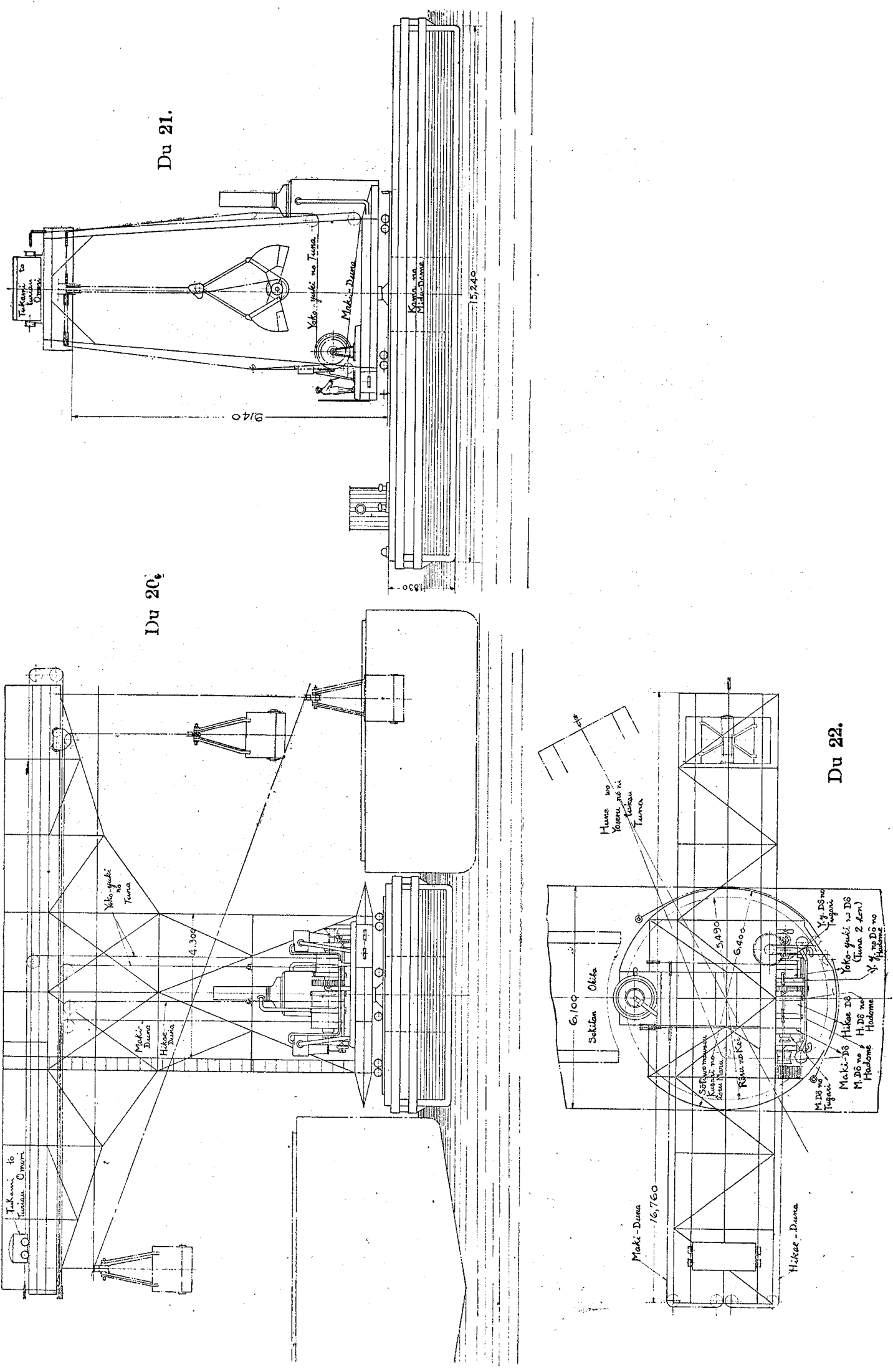




\begin{abstract}
Kono Baai niwa (Kikai wo mawasu koto wa betuni site)
\end{abstract}

Handoru no Kazu . . . 7 Handoru wo toru Kazu ............12

Handoru wo ugokasu Kazu ........19

Enzin no mawari-hazimeru Kazu....... 3

§ 17. Môtoru-mawasi no Sekitan Miduage Sôti. ${ }^{(5)}$ (Rei 2)

Kore wa Du $24 \sim \mathrm{Du} 26$ no Utusie ya $\mathrm{Du} \quad 27 \sim \mathrm{Du} \quad 29 \mathrm{ni}$ simesu yôni 1.1 ton no 1-ponduna-gata no Tukami de Sekitan wo Kisiage suru Sikake de Gendôki wa Tyokuryû $220 \mathrm{~V}$ no Siirisu-gata no Môtoru de aru.

Tukami wa $\$ 14$ no 1) no Baai no yôni ittan makiage te kara yokoni yuki Hoppâ no ue de Sekitan wo otosu, mata, kono tairana Tôrimiti ga migi-hidarini Kubi wo huru koto mo deki Hune no doko nimo Tukami wo otosu koto ga dekiru. (Kono hô wa koko dewa kangaenai.)

Tukami wo ugokasu Kikai wa mawareru Tôrimiti no ueni Hune to hantaino Grawa ni atte, Maki-Dô yoko-yuki no Dô, Turiai-Omori no Dô, nado no Hadome ya Tugari wa subete Denki no Magunetto wo motii, Untensyu ga zikani kono Handoru wo toru koto wa naku, kansetuni Denryû wo kittari iretari suru 1-ponno Handoru ga Untensyugoya ni aru bakari de aru.

Konohoka Maki-no-Môtoru no Kontorôrâ no Handoru ga 1-pon aru.

Du 30 wa Môtoru no Kontorôrâ no Sikumi wo, mata, Du 31 wa Hadome ya Tugari ni tukau Suitti no Sikumi wo simesu. ${ }^{(6)}$ Korera wa mina watakusi no atarasii Kôan de aru. Kono Sikake no Hatarakikata,

(5) Tsy. $13 \mathrm{n} .11 \mathrm{gt}$. dekiagaru.

(6) (Kono Sikake ni tuketa Tcriai-Omori ni tuitewa $\$ 11$ ni tokiakasita) 


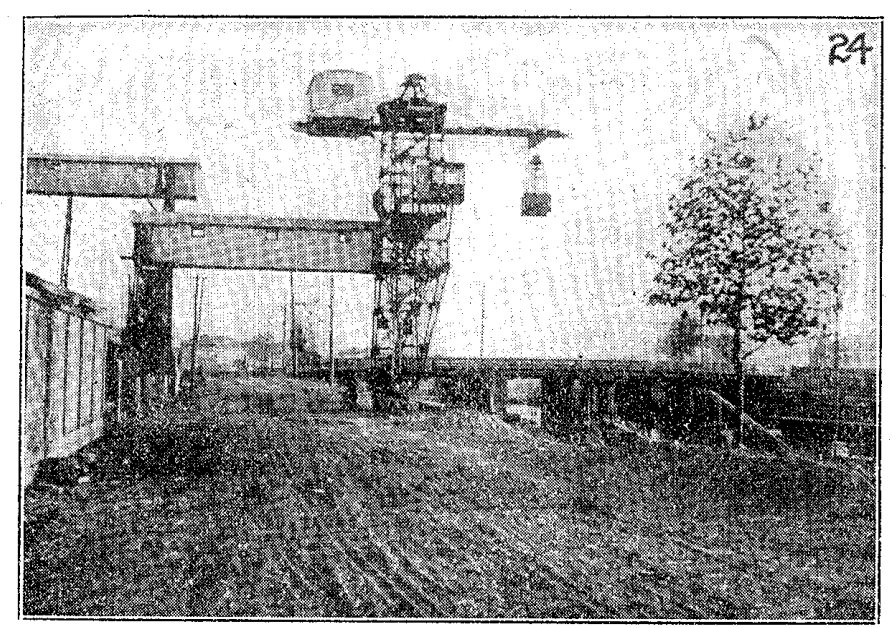

Du 24.

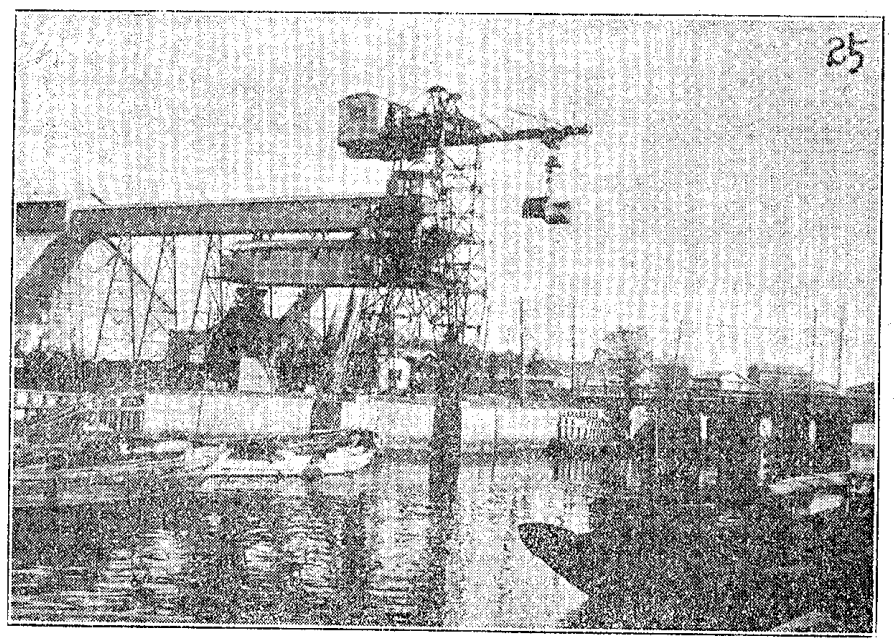

Du 25.

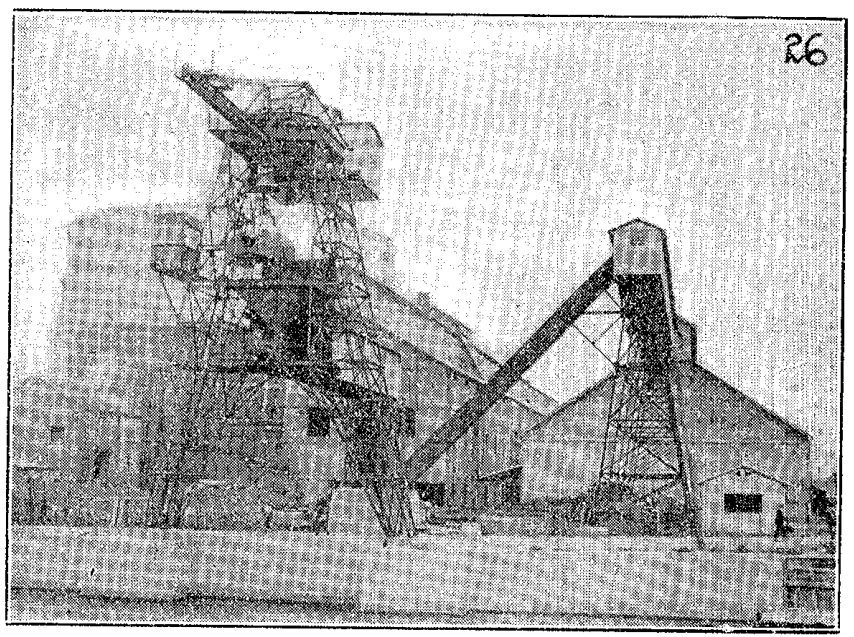

Du 26. 
Du 28.

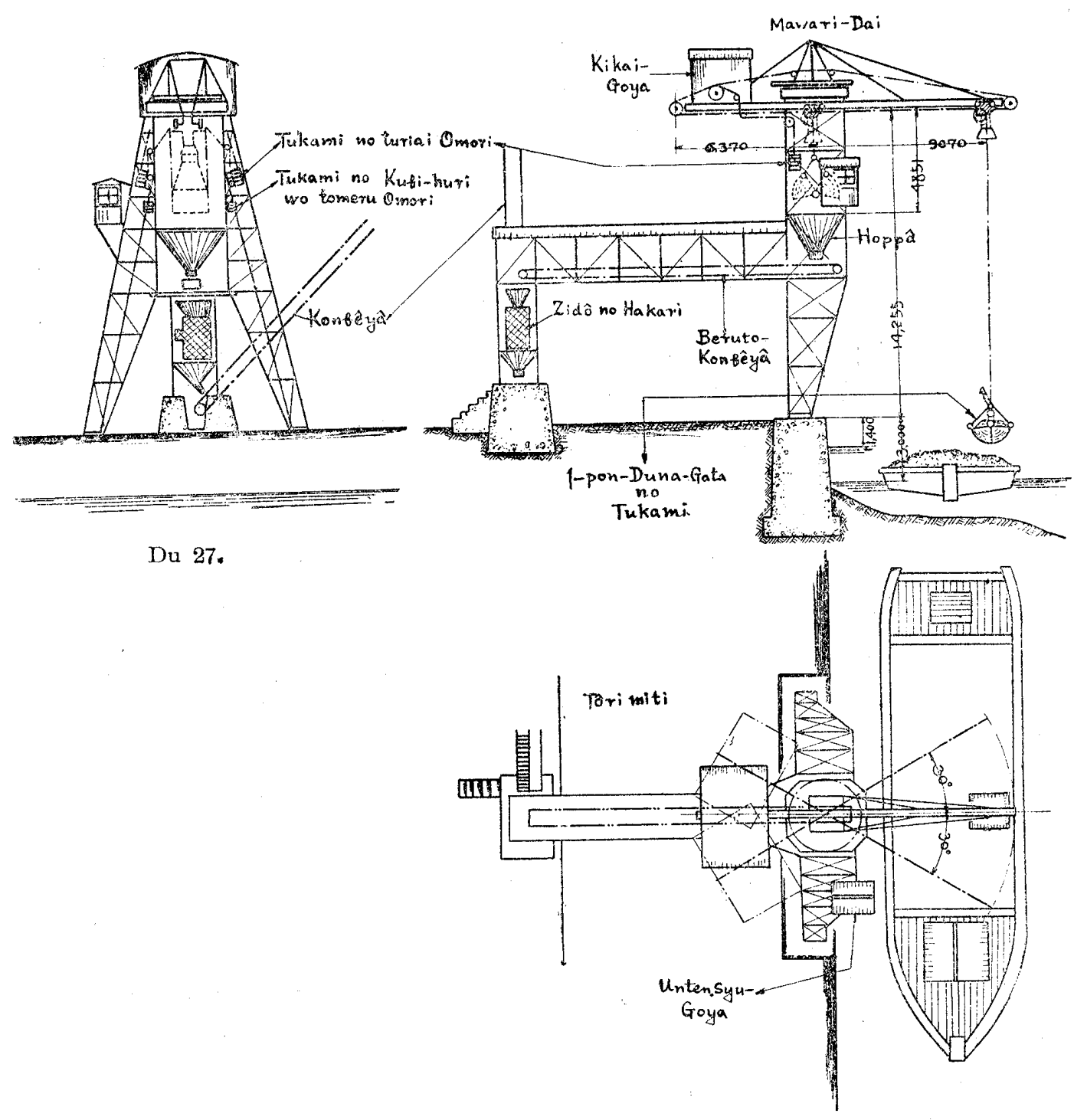

Du 29.

Hataraki ni iru Toki, itta Denryû nado wo hitomeni simesita mono ga Du 32 de' aru. Kono Baai ni (Kubihuri no Ugoki wa habulsu) wa, Handoru no Kazu.................. 2

Handoru wo toru Kazu . . . . . . . . . . . . . . 16

Handoru wo ugokasu Kazu................24

Môtoru no mawarihazimeru Kazu .......... 8

Handoru wo ugokasu Kazu towa Kontorôrầ no Handoru wo ippôni mawasu no ga 1 mata, sibaraku site Moto ni kaesu no ga 1 a wasete 2 to iu hûni kazoete 24 ni naru. Handoru wo toru Kazu no hô wa 
tatoeba kono Baai wa Kontorôrâ no Handoru wo 1-do totta to site kazoete 16 to naru no de aru.

Tukami ga yokoni yuku tokiniwa Môtoru ni kakaru Ni ga sukunai kara waduka $6 \mathrm{~m}$. kurai wo yuku no ni hayaku narisugite komaru kara Teikô wo ireta tokoro de mawasu no de aru ga, yahari, tometa tokoro de Tukami ga puran-puran hurete Tukami no Kagi ga Turigane kara hazuresô de komaru node Du nimo simesu yôni sibaraku mataneba naranu.

Zissaini hakatta Kekkwa ni yori $\$ 15$ no yôni $t$ wo kakiageruto,

$$
\begin{aligned}
t & =T_{t}+U_{t}+H_{t}+E_{t} \\
& =(3+7)+(17 \cdot 5+14 \cdot 5+6+6)+12+6 \\
& =10+44 \quad+12+6=72 \text { byô..... to naru }
\end{aligned}
$$

Sekkei no hazimeniwa nakanaka ôi. (Du 32) Zikken-siki. Tukue no ueno Kûron kara 60 byô nara zyûbun ni 1Hataraki wo yareru to omotta ga, zissai dekite miruto hazimewa 150 byô mo kakatta. Iroiro ni Handoru no Kata, Kontorôrâ no Kata ya Unten no Zyunzyo wo kangae yôyaku kokomade kogituita no de atta. Kono 72 byô yori mizikai toki mo aru ga nagai toki mo

\$18. Watakusi no

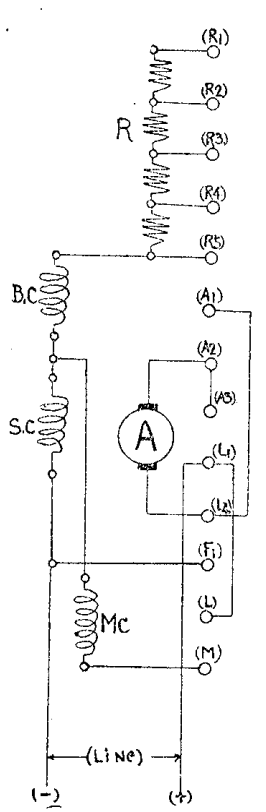

Korera no Rei no

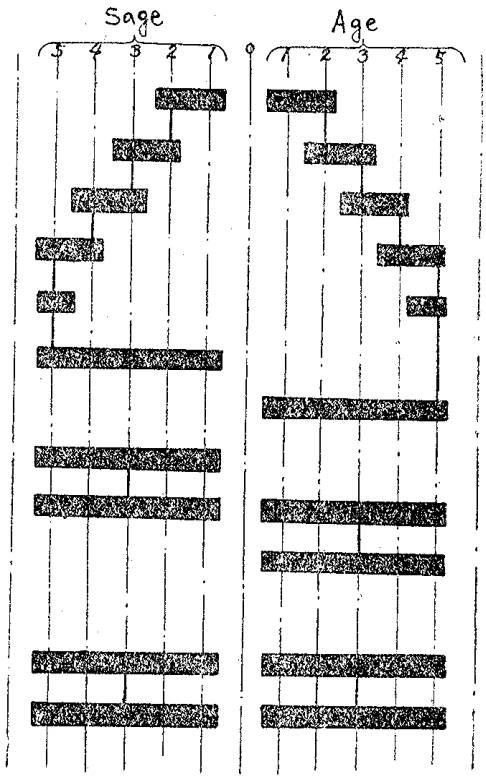

Du 30.

Zikkentekino Kangae wo ikuraka rikutu-dukete, watasi wa $t$ no $\mathrm{Ne}$ wo motomeru Zikken-siki wo tukutta.

$$
\begin{aligned}
t & =T_{t}+U_{t}+H_{t}+F_{t} \\
U_{t} & =\sum\left(\frac{60+S}{v}+t^{\prime}\right)
\end{aligned}
$$

$s \ldots \ldots \ldots \ldots$ I-ugoki no Nagasa .............m.

$S \ldots \ldots \ldots$. . ugoku Nagasa wo kuwaeawaseta mono ... m. 

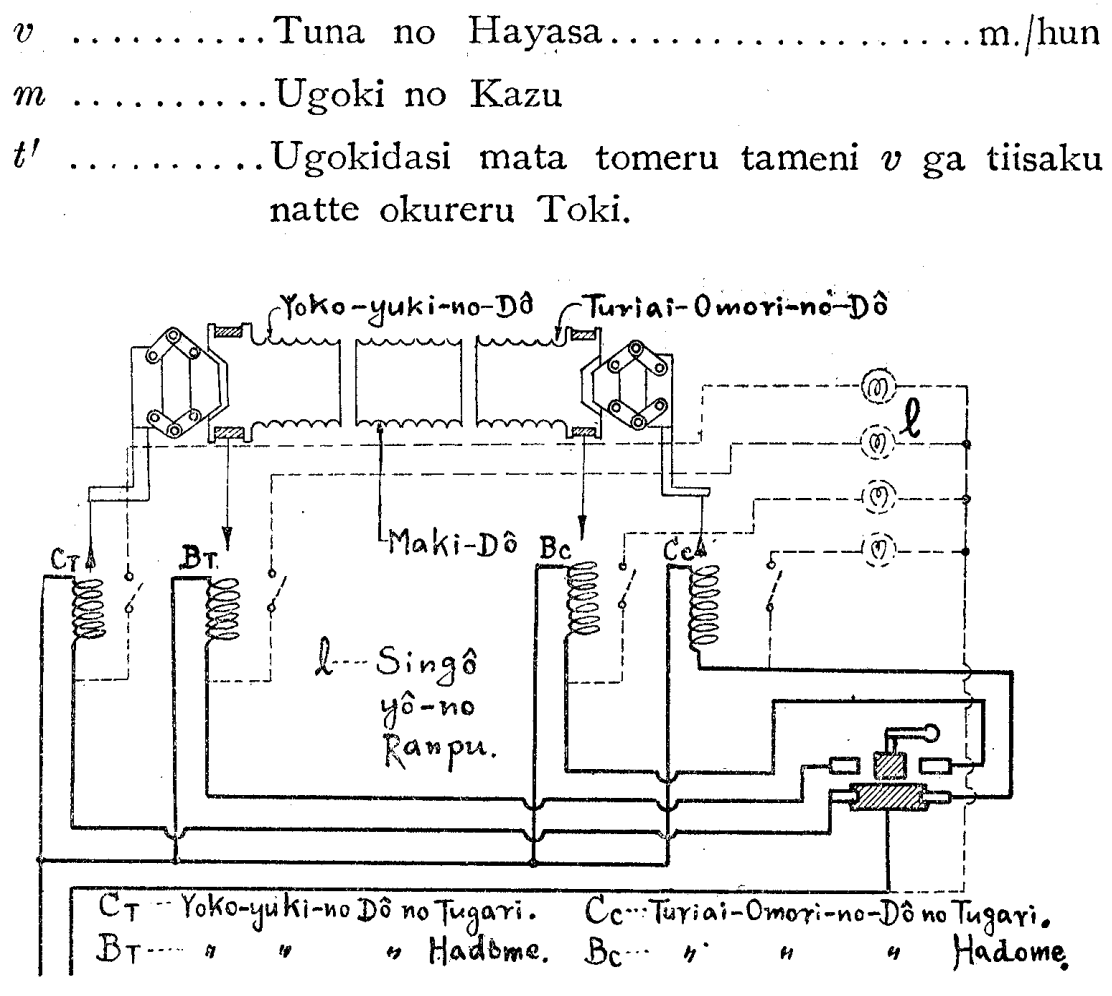

Du 31.

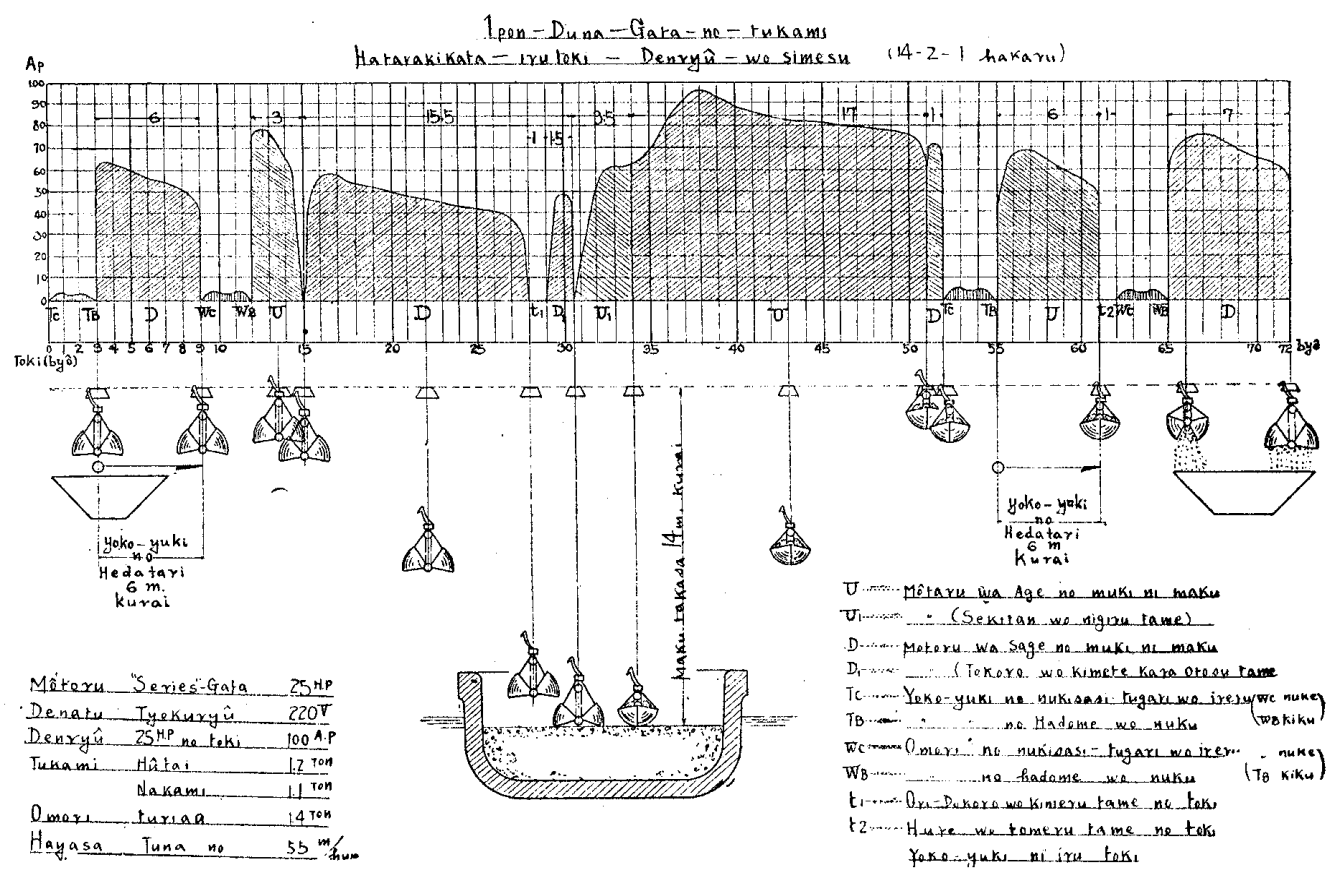

Du 32. 
Mosi onoonono Hayasa ga onazi de........v de areba,

$$
\begin{aligned}
& U_{t}=\frac{60 S}{v}+m t^{\prime} \\
& H_{t}=\Sigma t_{h} \\
& n^{\prime} \ldots \ldots \ldots \text { Handoru wo toru Kazu. } \\
& t_{t_{h}} \ldots \ldots \ldots \text {-do Handoru wo toru ni iru Toki. }
\end{aligned}
$$

Ima $t_{h}$ ga hitoiro da to suruto,

$$
\begin{aligned}
& H_{t}=n^{\prime} t_{h} \\
& t=T_{t}+\left(\frac{60 S}{v}+m t^{\prime}\right)+n^{\prime} t_{h}+F_{t}
\end{aligned}
$$

Kono Siki ni aru $T_{t}, t_{h}{ }^{\prime}, t_{h}$ oyb. $F_{t}$ no Ne wo watakusi wa tugino yôni kimeta.

Hyô 1. $T_{t}$ (byô)

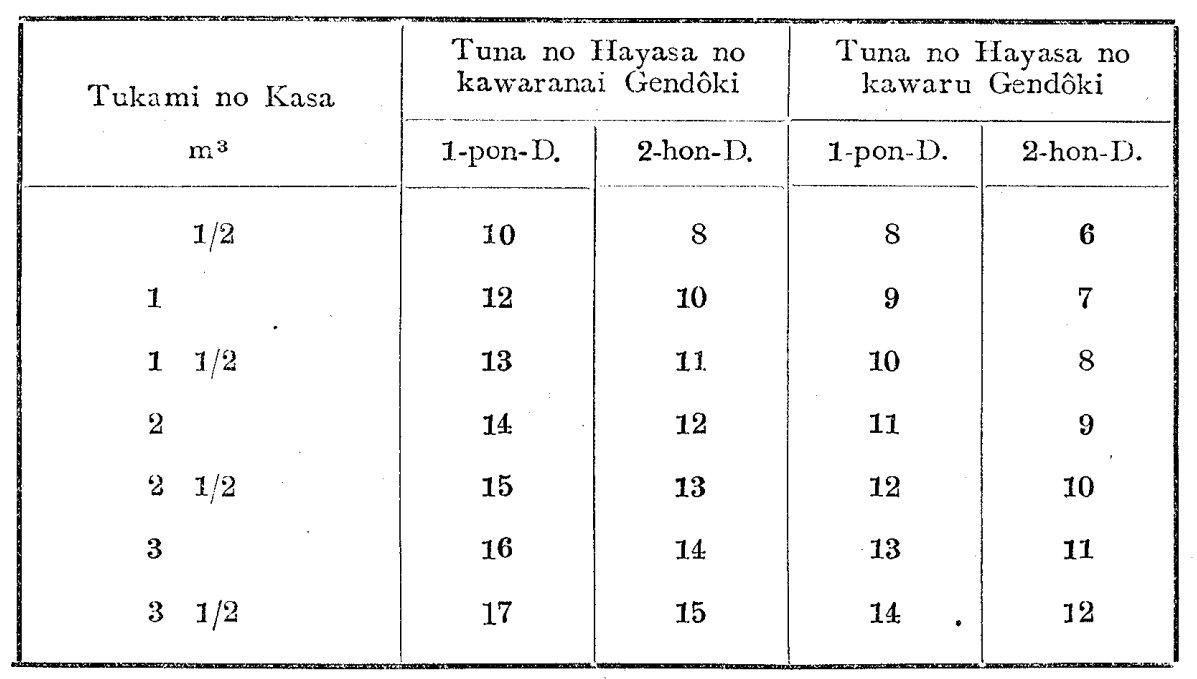

Tuna no Hayasa no kawaru Gendôki tatoeba Tyokuryû no Siirisu Môtoru toka Zyôki-Enzin no Baai niwa tukamu toki hazime Teikô no nai mata, sukunai toki kiwamete hayaku sosite dandanto Tukamizimai ni natte Tikara ga unto iru koro niwa osoku naru node Tugô ga yoi, keredomo, Kôryû no Indakusyon Môtoru no yôna Baai niwa Hayasa ga ittei de aru kara kono. Tukami-komi ni nakanaka Toki ga kakaru. Mata, 1-pon-duna no hô ga 2-hon-duna no hô yori Tuna no maku Nagasa ga masu node Toki ga kakaru. 
Hyô 2.

\begin{tabular}{|c|c|c|}
\hline & 1-pon-Duna & 2-hon-Duna \\
\hline$t^{\prime}$ byô & 1 & 1 \\
$F_{t}$ byô & $8 \sim 4$ & $3 \sim 0$ \\
\hline
\end{tabular}

Hyô 3.

\begin{tabular}{|c|c|c|}
\hline & $\begin{array}{c}\text { Tikara no iru Handoru } \\
\text { no Baai }\end{array}$ & $\begin{array}{c}\text { T ara no iranai Han- } \\
\text { doru no Baai }\end{array}$ \\
\hline$t_{h} \quad$ byô & $\mathbf{2} \sim \mathbf{1}$ & $\mathbf{0 7 5 \sim 0 . 5}$ \\
\hline
\end{tabular}

Tikara no iru Handoru to iu-nowa Hadome ya Tugari wo Te-de ugokasu yôna Baai de Kontorôrâ no Handoru nado wa Tikara no iranai hô ni kangaeru.

Siki (5) ni yotte maeni simesita 2 no Rei wo sirabete miru.

$\$ 16$ ni kaita Rei dewa,

$$
\begin{aligned}
& t=8+\left(\frac{60 S}{v}+4 \times 1\right)+12 \times 1 \cdot 5+3 \\
& t=8+\left(60 \frac{40}{120}+4\right)+18+3 \\
& t=8-(20+4)+18+3=53 \ldots \ldots \ldots \ldots \text { byô } \\
& \quad 68 \text { kwai } / \mathrm{zi} \ldots \ldots \ldots \ldots \ldots \text { to naru. }
\end{aligned}
$$

Tukami no Kasa ga 1-ton da kara

Nôryoku............

Mata, $\$ 17$ ni kaita Rei dewa

$$
\begin{aligned}
t= & 10+\left(60 \times \frac{40}{50}+7 \times 1\right)+16 \times 0 \cdot 75+6 \\
= & 10+51+12+6=79 \ldots \ldots \ldots \ldots \text { byô } \\
& 46 \mathrm{kwai} / \mathrm{zi} .
\end{aligned}
$$

Tukami no Kasa ga $\mathbf{1} \mathbf{1}$ da kara

. Nôryoku .............N=50 ton/zi.

to naru. (Owari)

Kono Sirabe wa watakusi ga K.-K.-Arakawa-Seisakusyo no Gisityô to site, mata sononoti hikituduite, Niyakukikai-Kenkyûsyo no Aruzi to site nasita mono de aru. ArakawaS. no Gisi de atta Kgs. Ban-K. Udi Kgs. Yano-M. Udi oyb. \$6 ni simesita Mokuromi ni tuite Otetudai wo negatta Niyakuk.-K. no Gisi Tubono-H. Udi nado ni taisite atuku Orei wo noberu.

Tsy. 15 n. 6 gt. 25 nt. Niyakukikai-Kenkyûsyo nite Nagawo-S. sirusu. 